\title{
Analysis and Design of Robust Quasilogarithmic Quantizer for the Purpose of Traffic Optimization
}

\section{Danijela Aleksić}

Telekom Srbija, Voždova 11-13, 18000 Niš, Serbia, e-mail: danijelaal@telekom.rs

\section{Zoran Perić}

University of Niš, Faculty of Electronic Engineering, Department of Telecommunications,

Aleksandra Medvedeva 14, 18000 Niš, Serbia, e-mail: zoran.peric@elfak.ni.ac.rs

Corresponding author: danijelaal@telekom.rs

This paper proposes a simple manner to determine the support region of the quasilogarithmic robust quantizer. We assume Laplacian probability density function which is widely accepted as a typical model for audio and speech signals in many applications in science and engineering. Theoretically, the adaptability and robustness are seemingly conflicting properties, while the numerical results point out at the fact that the proposed approach to the observed support region threshold determination provides good results in a wide dynamic range from the standpoint of the signal to quantization noise ratio (SQNR). Additionally, we propose an iterative method for support region determination, which stops when further improvement in mean-squared error (MSE) becomes negligible. The suggested model is useful for compression schemes that involve trade-offs between quantizer design and implementation complexity.

KEYWORDS: support region threshold, traffic optimization, robustness.

\section{Introduction}

PSTN (Public Switched Telephone Network) has been initially developed for circuit-switched traffic, providing a guaranteed connection between the source and the destination. Moreover, acceptable delay threshold is controlled through strict bandwidth allocation with each voice stream. The IP network, as originally designed data network, is additionally adapted to carry real-time voice traffic that requires a certain QoS (quality of service). In packet-switched communication systems, packets may be delayed or even lost during transmission. Configuring voice in a data network environment implies that voice packets are treated in such a way to withstand an acceptable amount of delay, jitter and packet loss. This is not crit- 
ical in most applications since the receiving end can request retransmission of the packet in question.

It is well known that in a real-time constrained and latency sensitive application, such as VoIP, highly stringent delay requirements are imposed. Accordingly, the retransmission is not feasible since this would introduce a considerable delay prohibiting a proper two-way conversation. Thus, lost and delayed packets must be compensated for at the receiving end. Although a great number of quantizers have been developed to provide an additional QoE (quality of experience) management, especially for the VoIP applications, where packet loss concealment algorithm is used [9], there is still the need to continue with research in this field.

Nowadays we are facing with the smart city [19] as a technology for a population, vehicles and other urban factors all of which are fused into a whole. It is obvious that the data traffic generated by various services and application grows rapidly, which is becoming a major challenge in network architecture. $5 \mathrm{G}$ will bring new interactive and immersive experiences to customers, who will continue to expect new services such as augmented reality-based applications, built on high-speed and low-latency communication, with imperceptible resulting delay, jitter or interruptions $[6,4]$. With more facilities being constantly added for an increased QoE (quality of experience), modern applications rely heavily on the cloud for a support [3].

Beyond that, the increasing demands of user applications have surged drastically and pulled up the explosive data traffic, at the same time requiring an efficient network structure to handle the data traffic all along. As the solution, offloading requires the help of some alternative networks to complement $[15,14]$. Thus, transmission networks are becoming increasingly heterogeneous [12]. Such systems often require each node to sustain a certain throughput demand. It is essential to determine a proper transmission rate in order to guarantee the system performance that can meet the application requirement and compensate for the network imperfections. Such a tuning in a heterogeneous network is difficult due to the lack of modeling techniques, which can handle with the network traffic changes. Numerous models of quantizers have been proposed aiming at the highest possible quality of the quantized speech signals along with the highest possible compression.
In the latest years, the Internet of Things (IoT) [22] is a model including ordinary entities with the ability to communicate with the corresponding devices using the Internet. There are a lot of research papers focusing on the complexities around the IoT [2] and Fog Computing, which extends the cloud closer to the things while processing the data generated by the devices. In general case, a Fog node can be any device with network connectivity, computing and storage capability, which can communicate with a variety of devices, offering a more efficient and quicker access.

In addition, a further insight in the importance of the sociological aspect emphasizes the need to collect, analyze, as well as to understand the relevant background of sociological processes. Non-verbal sounds such as laughing, crying, whispering, screaming, sneaking and sighing, which do not have to be accompanied by words, can provide additional information about the individual's attitude to a certain sociological interaction. There are many research papers which consider different communication challenges focusing on perceptual and bodily aspects of life in the modern world [13].

It is hard to predict what direction coding will take in the future. Instead of making coding obsolete, the interest in coding remains high nowadays.

Especially considering modern Man-Machine communication, we are trying to find easy and comfortable quantization method for interaction based on speech communication. Although effective integration of speech into man-machine communication depends on the nature of the user interaction and application, it is preferable to minimize the influence of non-verbal behavior and the difficulties appearing in emotional or sociological background during voice communication.

Promising discretization of any kind of input signal vindicate undeniably extremely important role of the quantization process in overall digital signal processing. While never without diminishing importance different quantizer models promise innovative solutions to the global challenges of discretization task. In this paper, we propose an efficient and low-complexity quantizer design. The high level of adaptability to the signal variance helps ensure that suggested robust quantizer can facilitate further performance estimation and optimization. 
The paper is structured as follows. Section 2 provides a descriptive and detailed simple solution to the problem of determining the support region of the quasilogarithmic quantizer, designed for the Laplacian source and an arbitrary variance. Section 3 is devoted at providing the numerical results and the topic. Finally, related conclusions, which are summarizing the contribution achieved in the paper, are presented in Section 4.

\section{Support Region of the Quasilogarithmic Quantizer Designed for the Laplacian PDF and an Arbitrary Variance}

The term quasi-static signal refers to a signal that changes so slowly over a long time so that it acquires characteristics more like static signals than dynamic signals [11]. The network environment requires very flexible coders that are able to adjust in real time to continuously changing network conditions, and particularly to varying transmission rate. In a multirate system where narrowband and wideband speech are supported, G.711 and G.722 codecs are used simultaneously.

The selection of the compression of signals and quantization method is a compromise between the highest possible perceived quality of signals for the given number of bits or, conversely, minimizing the number of bits required to encode a signal at a given quality. The various compression techniques offer different levels of complexity, compressed signal quality and the amount of compressed data. It is well known that G.711 $[8,10]$ is a coding standard for narrowband speech and that it works on the principle of companding. The codec uses $A / \mu$-law companding technique to encode and decode speech.

Quasilogarithmic quantizer represents a logarithmic quantizer defined with the $\mu$ compression law. It is well known that, according to the G.711 coding standard, compression factor is equal to 255 [11]. Additionally, in this paper we assume various compression factors for which we analyze the performance of the proposed encoding/decoding algorithm, coupled with G.711.

In what follows, we assume that the signal at the input of the quantizer originates from a Laplacian source.
For a quasilogarithmic quantizer $Q_{\mu}{ }^{N}$ designed for a variance $\sigma_{p}^{2}$, where $\mu$ is the compression factor and $N$ is the number of quantization levels, compression is performed using the $\mu$-law compressor function $c_{\mu}(x)$ : $\left[-x_{\max }^{\sigma_{p}}, x_{\max }^{\sigma_{p}}\right] \rightarrow\left[-x_{\max }^{\sigma_{p}}, x_{\max }^{\sigma_{p}}\right][11,7]$ :

$c_{\mu}(x)=\frac{x_{\max }^{\sigma_{p}}}{\ln (1+\mu)} \ln \left(1+\mu \frac{|x|}{x_{\max }^{\sigma_{p}}}\right) \operatorname{sgn}(x)$,

for $|x| \leq x_{\max }^{\sigma_{p}}$, wherein the $x_{\max }^{\sigma_{p}}$ stands for the $\mu$-law companding quantizer's support region threshold.

For a given quantizer model wherein $\left[-x_{\max }^{\sigma_{p}}, x_{\max }^{\sigma_{p}}\right]$ defines the $\mu$-law companding quantizer's support region, the expression for the total distortion is given by [1]:

$$
\begin{aligned}
D\left(Q_{\mu}^{N}\right) & =C \sigma_{p}^{2}\left[\frac{1}{\mu^{2}} \frac{x_{\max }^{\sigma_{p}^{2}}}{\sigma_{p}^{2}}+\frac{x_{\max }^{\sigma_{p}}}{\sigma_{p}} \frac{\sqrt{2}}{\mu}+1\right], \\
& +\sigma_{p}^{2} \exp \left(-\frac{\sqrt{2} x_{\max }^{\sigma_{p}}}{\sigma_{p}}\right)
\end{aligned}
$$

where $C=\ln ^{2}(\mu+1) /\left(3 N^{2}\right)$ is a constant.

An analysis given in [11] uncovers several insights on the relationship between granular, overload and total distortion. Additionaly, in [1], a general framework is provided for interpreting the impact of both granular and overload distortion to the number of quantization levels and the support region width.

Indeed, an $N$-level quantizer will be said to be globally optimal if it minimizes the expected distortion. An approach that includes estimation and optimization for a quantizer's support region and the number of quantization levels, has proved as quite useful for designing scalar quantizers with known distributions, that were sufficiently well behaved to ensure the existence of minimal distortion [17,16]. In fact, one heuristic solution for the support region of the optimal and asymptotically optimal fixed-rate scalar quantizers has already been shown in [17]. In addition, paper [16] deals with the upper bound of this support region.

A lot of quasilogarithmic quantizers were shown to be applicable to solve for the minimum distortion. In $[18,20]$ some solutions to the two-stage quantization model based on quasi-logarithmic quantizers are giv- 
en. The prior work [21] that included backward adaptation technique, has offered a complex but improved solution to the quasilogarithmic quantizer model, preferable for the smaller compression factor values. The source coding in [5] adopts frame-by-frame processing using the combination of two three-level restricted quantizers, while taking into consideration the unequal support regions, selected according to the lower distortion criteria.

An intention of this paper is to generalize support region estimation by giving an expression for the maximal signal amplitude and its numerical computation. Furthermore, our objective is to extend the analysis of support region $x_{\max }^{\sigma_{p}}$ determination to the cases where the compression factor $\mu$ has an arbitrary value. If we assume that the value of the parameter $\mu$ is large enough (for instance $\mu=255$ as defined by G.711 standard), we obtain the following approximate formula for total distortion $D^{L}\left(Q_{\mu}{ }^{N}\right)$ :

$$
\begin{aligned}
& D^{L}\left(Q_{\mu}^{N}\right)=D\left(Q_{\mu=255}\right)= \\
& =C \sigma_{p}{ }^{2}\left[\frac{x_{\max }^{\sigma_{p}}}{\sigma_{p}} \frac{\sqrt{2}}{\mu}+1\right]+\sigma_{p}^{2} \exp \left(-\frac{\sqrt{2} x_{\max }^{\sigma_{p}}}{\sigma_{p}}\right) .
\end{aligned}
$$

In accordance with the minimal distortion criteria, achieved by setting the first derivate of the so obtained distortion $D^{L}\left(Q_{\mu}{ }^{N}\right)$ to zero with respect to $x_{\max }^{\sigma_{p}}$ :

$\frac{\ln ^{2}(1+\mu)}{3 N^{2}} \frac{1}{\mu}-\exp \left\{-\frac{\sqrt{2} x_{\max }^{\sigma_{\mathrm{p}}, \mathrm{L}}}{\sigma_{p}}\right\}=0$,

the support region threshold $x_{\max }^{\sigma_{p}, L}$ is derived as:

$$
x_{\max }^{\sigma_{\mathrm{p}}, \mathrm{L}}=\frac{\sigma_{p}}{\sqrt{2}} \ln \left(\frac{3 N^{2} \mu}{\ln ^{2}(1+\mu)}\right) .
$$

According to minimal distortion criteria for the total distortion given by $D\left(Q_{\mu}{ }^{N}\right)(3)$ :

$$
\frac{2 C x_{\max }^{\sigma_{p}}}{\mu^{2}}+\frac{C \sqrt{2}}{\mu}-\sqrt{2} \exp \left(-\sqrt{2} x_{\max }^{\sigma_{p}}\right)=0 .
$$

In order to determine $x_{\max }^{\sigma_{p}}$ the application of iterative numerical method is required:

$$
\begin{aligned}
x_{\max }^{\sigma_{p}{ }^{(i)}} & =\frac{\sigma_{p}}{\sqrt{2}} \ln \left(\frac{3 N^{2} \mu}{\ln ^{2}(1+\mu)} \frac{1}{1+\frac{\sqrt{2}}{\mu} \frac{x_{\max }^{\sigma_{p}}}{\sigma_{p}}}\right) \\
& =\frac{\sigma_{p}}{\sqrt{2}} \ln \left(\frac{\mu}{C} \frac{1}{1+\frac{\sqrt{2}}{\mu} \frac{x_{\max }^{\sigma_{p}{ }^{(i-1)}}}{\sigma_{p}}}\right) .
\end{aligned}
$$

To accelerate the estimation of $x_{\max }^{\sigma_{p}}$, we initialize the iterative method using the support region threshold obtained in (5):

Step1. The initialization of the algorithm:

$$
\begin{aligned}
& x_{\max }^{\sigma_{p}{ }^{(0)}}=x_{\max }^{\sigma_{p}, L} \\
& \left.x_{\max }^{\sigma_{p}{ }^{(1)}}\right|_{x_{\max }^{\sigma_{p}(0)}=x_{\max }^{\sigma_{p}, L}}=\frac{\sigma_{p}}{\sqrt{2}} \ln \left(\frac{\frac{\mu}{C}}{1+\frac{1}{\mu} \ln \left(\frac{\mu}{C}\right)}\right),
\end{aligned}
$$

where $C=\ln ^{2}(\mu+1) /\left(3 N^{2}\right)$ is a constant.

Step2. The computation of new threshold value $x_{\max }^{\sigma_{p}}(2)$ using (7) and the interruption of the iterative method if the estimated relative error defined as:

$$
\delta[\%]=\left|\frac{x_{\max }^{\sigma_{p}(2)}-x_{\max }^{\sigma_{p}(1)}}{x_{\max }^{\sigma_{p}(2)}}\right| \times 100
$$

is less than $5 \cdot 10^{-3}$.

\section{The Numerical Results}

In this section, our objective is to ascertain and summarize the performances for quasilogarithmic quantizer for an arbitrary variance in a wide variance range. Here, by identifying $x_{\max }^{\sigma_{p}}=k \sigma_{p}$, according to (5) and (8), one can come to the conclusion that $x_{\max }^{\sigma_{p}}$ can be presented by using multiplying coefficient $k$ :

$$
x_{\max }^{\sigma_{p}}=k \sigma_{p},
$$




$$
k=\left\{\begin{array}{l}
\frac{1}{\sqrt{2}} \ln \left(\frac{3 N^{2} \mu}{\ln ^{2}(1+\mu)}\right), \text { for } x_{\max }^{\sigma_{p}, L} \\
\frac{1}{\sqrt{2}} \ln \left(\frac{\frac{3 N^{2} \mu}{\ln ^{2}(1+\mu)}}{1+\frac{1}{\mu} \ln \left(\frac{3 N^{2} \mu}{\ln ^{2}(1+\mu)}\right)}\right), \text { for } x_{\max }^{\sigma_{p}(1)}
\end{array} .\right.
$$

In order to account for the deployment of quasilogarithmic quantizer and arbitrary variance $\sigma_{p}^{2}$ in a wide dynamic range of input variance $\sigma_{q}^{2}$, we assume that the design for the quasilogarithmic quantizer for an arbitrary variance $\sigma_{q}^{2}$ is based on the design of the same quantizer for the $\sigma_{p}^{2}$ along with the scaling with the coefficient $\rho$ :

$$
\begin{aligned}
& \rho=\sigma_{q} / \sigma_{p} \\
& D\left(Q_{\mu}^{N}\right)= \sigma_{q}^{2} \frac{\ln ^{2}(1+\mu)}{3 N^{2}}\left(\frac{k^{2}}{\mu^{2} \rho^{2}}+\frac{\sqrt{2} k}{\mu \rho}+1\right) \\
&+\sigma_{q}^{2} \exp \left\{-\frac{\sqrt{2} k}{\rho}\right\} .
\end{aligned}
$$

For a given quasilogarithmic quantizer's model, the minimum distortion criterion is employed to assure the maximum of SQNR:

$$
\begin{aligned}
& \text { SQNR }=10 \log _{10}\left(\frac{\sigma_{q}^{2}}{D\left(Q_{\mu}^{N}\right)}\right)= \\
& =-10 \log _{10}\left(C\left(\frac{a^{2}}{\mu^{2}}+\frac{\sqrt{2} a}{\mu}+1\right)+\exp \{-\sqrt{2} a\}\right)
\end{aligned}
$$

where $a=k / \rho$.

Figures 1-3 show the SQNR characteristics of the suggested quantizer for various compression factors.

It should be noted that for $\mu=10$ and $R=5$ or 6 , SQNR can range more than $15 \mathrm{~dB}$ for the range $\rho=0.2$ to $\rho=2$, while for $\mu=100$ and $R=3$ or 4 , SQNR can range more than $10 \mathrm{~dB}$ for $\rho=0.2$ to $\rho=2$. At higher bit rates, for example for $R=8$ and higher, for the range $\rho=0.2$ to $\rho=2$, SQNR higher than 20 or $30 \mathrm{~dB}$ can be obtained for $\mu=10$ or $\mu=100$, respectively. In this way, by selecting the parameter $\mu$ and $\mathrm{R}$, and in accordance with the

\section{Figure 1}

SQNR dependence on the scaling coefficient $\rho$ for $\mu=255$ and $R=3-8$ [bit/sample]

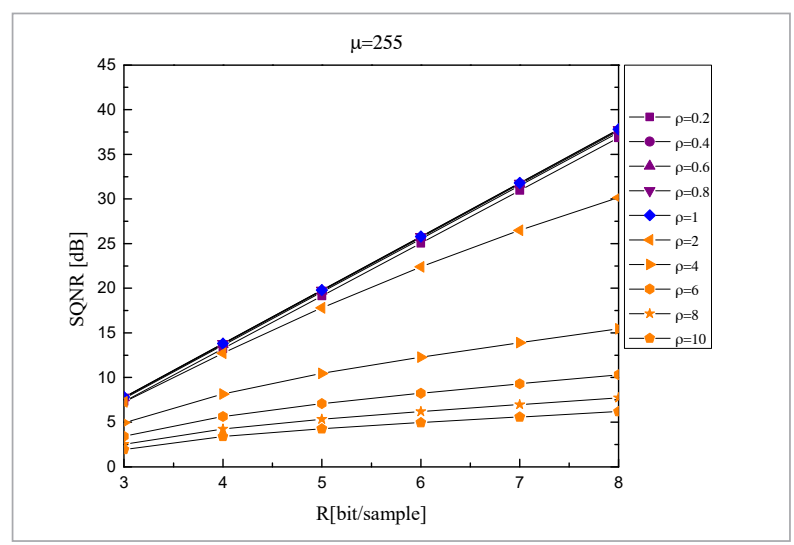

Figure 2

SQNR dependence on the scaling coefficient $\rho$ for $\mu=10$ and $R=3-8$ [bit/sample]

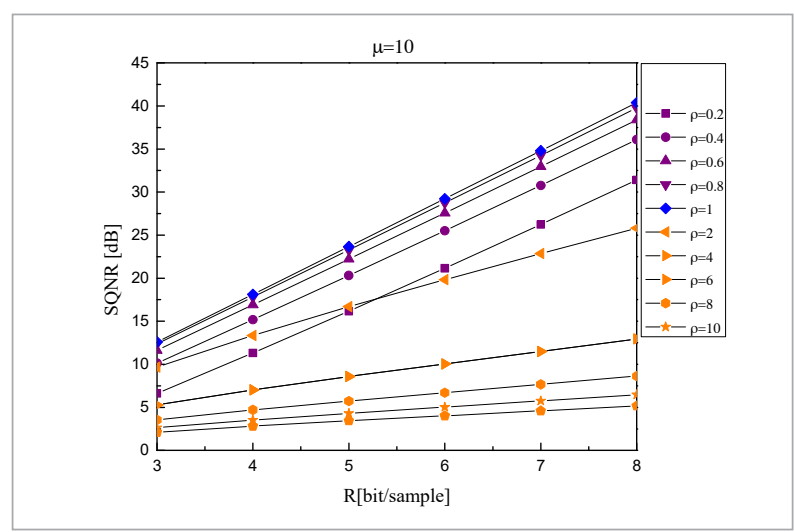

Figure 3

SQNR dependence on the scaling coefficient $\rho$ for $\mu=100$ and $R=3-8$ [bit/sample]

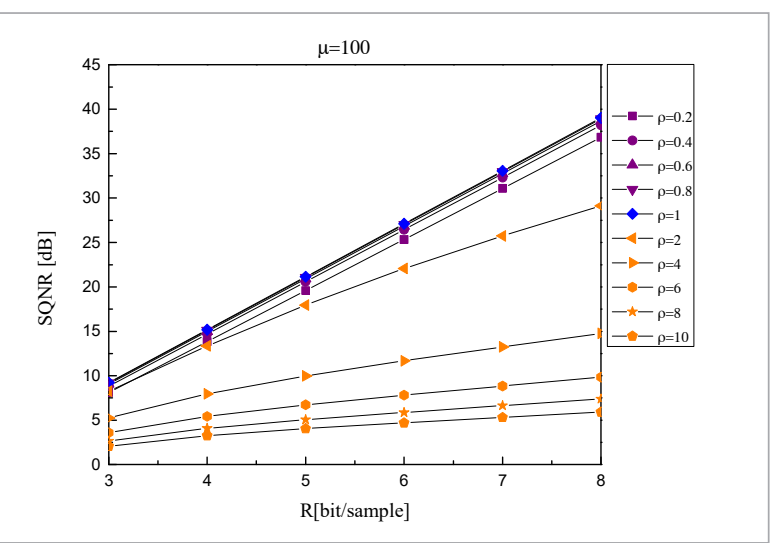


Table 1

Parametres for the analysis of the accuracy of the formula for the support region threshold of the quasilogarithmic quantizer

\begin{tabular}{|c|c|c|c|c|c|}
\hline Compression factor $\mu$ & R [bit/sample] & $x_{\max }^{\sigma_{p}}$ & $x_{\max }^{\sigma_{p}^{(1)}}$ & $x_{\max }^{\sigma_{p}}(0)$ & $\begin{array}{c}\delta \\
{[\%]}\end{array}$ \\
\hline \multirow{3}{*}{$\mu=255$} & 4 & 6.1699 & 6.1698 & 6.1937 & 0.0015 \\
\hline & 6 & 8.1230 & 8.1229 & 8.1542 & 0.0014 \\
\hline & 8 & 10.0763 & $10.0^{7} 61$ & 10.1147 & 0.0014 \\
\hline \multirow{3}{*}{$\mu=100$} & 4 & 5.7363 & 5.7357 & 5.7914 & 0.0089 \\
\hline & 6 & 7.6790 & 7.6783 & 7.7519 & 0.0086 \\
\hline & 8 & 9.6222 & 9.6214 & 9.7124 & 0.0083 \\
\hline \multirow{3}{*}{$\mu=10$} & 4 & 4.7285 & 4.7058 & 5.0892 & 0.4790 \\
\hline & 6 & 6.5856 & 6.5606 & 7.0497 & 0.3785 \\
\hline & 8 & 8.4552 & 8.4292 & 9.0102 & 0.3077 \\
\hline
\end{tabular}

required minimum values of SQNR and the variance range, the choice of the performances of the quasilogarithmic quantizer can be balanced, at the same time achieving additional optimization in the amount and volume of the transferred data in bits.

The proposed support region estimation method makes possible simple initialization of the suggested algorithm. Namely, the initial value $x_{\max }^{\sigma_{p}(0)}$ for the suggested iterative method is equal to $x_{\max }^{\sigma_{p}, L}=x_{\max }^{a}$, which is obtained from an approximate closed-form formula for the support region threshold of the quasilogarithmic quantizer designed for the Laplacian source of an arbitrary variance given in [10].

Moreover, one can notice that the suggested iterative method converges very fast. For the simplicity purpose, we can assume that determining $x_{\max }^{\sigma_{p}}$ is accomplished after only one itteration. In order to account for the assumption validation, the relative error of estimating the support region threshold is calculated for $\mu=255, \mu=100, \mu=10$ and a different number of quantization levels $N(N=16, N=64$ and $N=256)$, listed in Table 1 . according to (9).

Notably, the potential for the support region threshold determination with the reduced number of used bits can be highlighted. We address the problem of suitable support region threshold determination by limiting a relative error approximately to a value less than $0.5 \%$, in the worst case.

Here, we have shown that the SQNR of the considered quasilogarithmic quantizer is additionally tuned us- ing fast and accurate estimation of the support region threshold that provides minimal total distortion for the signal with an accommodated amplitude dynamic.

\section{Conclusion}

Our aim is to outline the main conclusion when the robust quantizer presents the underlying desirable solution out of the whole set of different quantizer's solutions.

To that end, we have evaluated the performance of robust quantizer without obligatory insisting on the highest SQNR values.

For the sake of clarity in the paper, we can admit that the obtained SQNR values for the suggested quasilogarithmic quantizer are noticeably lower compared to sub-band solutions presented in [21].

In fact, the performance appraisal requires that one quantizer solution is preferred over other solutions according to some relevant qualifiers.

It is well known that a lot of quantization models are suggested with the goal to minimize the number of used bits for signal storage and transmission while keeping, at the same time, an adequate signal quality level. Many applications specify different tradeoffs depending on the required signal quality levels. We want to outline an appropriate robust quantizer model as our proposal for the optimal speech and audio signal. 
Initially assuming that the speech signal can have unpredictable statistical characteristics, we accept that it is most appropriate to use quasilogarithmic quantizer, which provides robustness in a wide range of input signal variances. Although the $A / \mu$-law companding has become widely used as a design guideline for nonuniform quantization of speech signals, the fundamental question of how to provide a simple manner to optimize parameters of quantizer for signals with Laplacian probability density function has remained open.

Before it becomes a problem, a lot of congestion avoidance techniques monitor network traffic loads,

\section{References}

1. Aleksić, D., Perić, Z., Nikolić, J. Support Region Determination of the Quasilogarithmic Quantizer for Laplacian Source. Przeglad Elektrotechniczny, 2012, 88(7A), 130-132.

2. Atzori, L, Iera, A., Morabito, G., Nitti, M. The Social Internet of Things (SIoT) when Social Networks Meet the Internet of Things: Concept, Architecture and Network Characterization, Computers Networks, 2012, 56(16), 3594-3608. https://doi.org/10.1016/j.comnet.2012.07.010

3. Blažauskas, T., Muliuolis, A., Bikulčienè, L., Butkevičiūtè, E. Service-Oriented Architecture Solution for ECG Signal Processing. Information Technology and Control, 2017, 46(4), 445-458. https://doi.org/10.5755/ j01.itc.46.4.18470

4. Chen, S., Zhao, J. The Requirements, Challenges, and Technologies for 5G of Terrestrial Mobile Telecommunication. IEEE Communications Magazine, May 2014, 52(5), 36-43. https://doi.org/10.1109/ MCOM.2014.6815891

5. Denić, B., Perić, Z., Despotović, V., Vučić, N. Forward Adaptive Laplacian Source Coding Based on Restricted Quantization. Information Technology and Control, 2018, 47(2), 209-219. https://doi.org/10.5755/j01. itc. 47.2 .16670

6. GSMA Intelligence Understanding 5G: Perspectives on Future Technological Advancements in Mobile. White Paper, 2014, 6-13.

7. Hanzo, L., Somerville, C. A., Woodard, J. Voice and Audio Compression for Wireless Communications. John Wiley \& Sons - IEEE Press, 11-28, 2007. https://doi. org/10.1002/9780470516034

8. Hiwasaki, Y., Sasaki, S., Ohmuro, H., Mori, T., Seong, J., Lee, M. S., Kövesi, B., Ragot, S., Garcia, J.-L., Marro, C., especially for a prioritized traffic class, such as voice. It is worth emphasizing that the suggested quasilogarithmic quantizer model allows for a different bit allocation with respect to the requirements of the minimal desired SQNR conditions. It can facilitate the accurate anticipation of the traffic from the standpoint of the traffic optimization.

Additionally, the suggested quantization model and analysis can be directed both toward existing speech communication systems with logarithmic compandors, as well as toward forecasting of solutions, which will be available in the near future.

Miao, L., Xu, J., Malenovsky, V., Lapierre, J., Lefebvre, R. 5G.711.1: A Wideband Extension to ITU-T G.711. Proceedings of EUSIPCO'08, Lausanne, Switzerland, 2008.

9. ITU-T Recommendation G.711. Appendix I, A High Quality Low-Complexity Algorithm for Packet Loss Concealment with G.711, Sep. 1999.

10. ITU-T. Recommendation G.711.1, Wideband Embedded Extension for G.711 Pulse Code Modulation, 2008.

11. Jayant, N. S., Noll, P. Digital Coding of Waveforms. New Jersey, Prentice Hall, Chapter 5, 221-251, 1984.

12. Jo, H.-S., Sang, Y. J., Xia, P., Andrews, J. G. Heterogeneous Cellular Networks with Flexible Cell Association: A Comprehensive Downlink SINR Analysis. IEEE Transactions on Wireless Communications 11(10), 2012, 3484-3495. https://doi.org/10.1109/ TWC.2012.081612.111361

13. Kramer, A. D., Guillory, J. E., Hancock, J. T. Experimental Evidence of Massive-Scale Emotional Contagion Through Social Networks. PNAS, 2014, 111(24), 87888790. https://doi.org/10.1073/pnas.1320040111

14. Kumar, K., Liu, J., Lu, Y.-H., Bhargava, B. A Survey of Computation Offloading for Mobile Systems. Mobile Networks and Applications, 2013, 18(1),129-140. https://doi.org/10.1007/s11036-012-0368-0

15. Lee, K., Lee, J., Yi, Y., Rhee, I., Chong, S. Mobile Data Offloading: How Much Can WiFi Deliver?, IEEE/ACM Transactions on Networking, 2013, 21(2), 536-550. https://doi.org/10.1109/TNET.2012.2218122

16. Na, S. On the Support of Fixed-Rate Minimum MeanSquared Error Scalar Quantizers for a Laplacian Source. IEEE Transactions on Information Theory, 2004, 50(5), 937-944. https://doi.org/10.1109/TIT.2004.826686 
17. Na, S. Neuhoff, D. L. On the Support of MSE-Optimal, Fixed-Rate, Scalar Quantizers. IEEE Transactions on Information Theory, 2001, 47(7), 2972-2982. https:// doi.org/10.1109/18.959274

18. Perić, Z., Nikolić, J., Mosić, A., Panić, S. A Switched -Adaptive Quantization Technique Using $\mu$-Law Quantizers. Information Technology and Control, 2010, $39(4), 317-320$.

19. Skouby K. E., Lynggaard, P. Smart Home and Smart City Solutions Enabled by 5G, IoT, AAI and CoT Services. International Conference on Contemporary Computing and Informatics, 2014, 874-878. https://doi. org/10.1109/IC3I.2014.7019822
20. Tančić M., Perić, Z., Simić, N., Tomić, S. Performance of Quasi-Logarithmic Quantizer for Discrete Input Signal. Information Technology and Control, 2017, 46(3), 395402. https://doi.org/10.5755/j01.itc.46.3.16197

21. Tomić, S., Perić, Z., Tančić, M., Nikolić, J. Backward Adaptive and Quasi-Logarithmic Quantizer for Sub- Band Coding of Audio. Information Technology and Control, 2018, 47(1), 131-139. https://doi.org/10.5755/j01.itc.47.1.16190

22. Wolff, C., Knirr, M., Priebe, K., Schulz, P., Strumberg, J. A Layered Software Architecture for a Flexible and Smart Organic Rankine Cycle (ORC) Turbine - Solutions and Case Study. Information Technology and Control, 2018, 47(2), 349-362. https://doi.org/10.5755/ j01.itc.47.2.19681 